\title{
Allowing and Storing of Authorized and Unauthorized Database User According to the Policy Verification and Validation of Distributed Firewall under the Specialized Database
}

\author{
P.Senthilkumar \\ Lecturer-CSE \\ Nandha College of Technology \\ Erode
}

\author{
Dr.S.Arumugam \\ Chief Executive Officer \\ Nandha Engineering College \\ Erode
}

\begin{abstract}
The society has grown to rely on internet services, and the number of internet client increases every day. As more users are connected to the network, millions a user to do their damage becomes very great and lucrative. In conventional firewall rely on topology restrictions and controlled network entry points to enforce packet filtering. In this paper, I propose method of multiple firewall concepts and also maintain the database for both the authorized and unauthorized entry details based on security policy to enforce the static and dynamic packet filtering. This technique is implemented in software tool called distributed firewall policy advisor and specialized database (SDB).
\end{abstract}

Keywords- Firewall, Distributed Firewall, policy Language, policy verification, Policy validation, Specialized Database(SDB),Distributed firewall policy Advisor(DFPA).

\section{INTRODUCTION TO FIREWALL}

The firewall is a computer hardware or software that limits access to a computer over a network or from an outside source. The firewall is used to create security check points at the boundaries of private network. The general firewalls shows the figure 1.1

A firewall is placed at an entry point where a private computer network is connected to the outside Internet. It intercepts all the packets that are exchanged between the private computer network and the rest of the Internet and examines the IP, TCP and UDP headers of each intercepted packet and decides whether to accept the packet or to discard the packet network of a large enterprise has tens or even hundreds of firewalls. These firewalls are placed at the entry points of the private

In the case of companies, if when ordinary firewall is used everyone were given the same class policy. By the implementation of the distributed firewall, multiple firewall concepts each and every one with in the organization was provided with separate access policy, separate authentication. [12]

\subsection{General Techniques}

General techniques that firewall use to control access and enforce the site's security policy.

\section{Service control:}

It determines the types of internet service that can be accessed inbound (or) outbound.

\section{Direction control:}

It determines the direction in which particular service request may be indicate and allowed to flow through the firewall.

\section{User control:}

Control access to service according to which user is attempting to access it.

\section{Behavioral control:}

Controls now particular services are used.

\section{Firewall diagram}

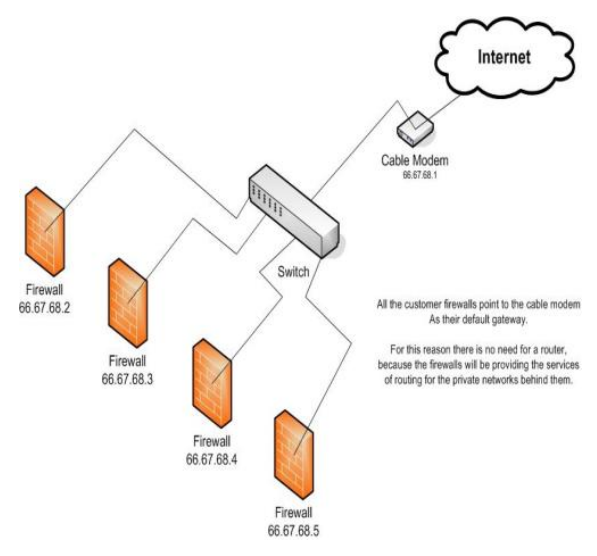

1.1 figure

\section{THE DISTRIBUTED FIREWALL}

A distributed firewall uses a central policy, but pushes enforcement towards the edges. That is, the policy defines what connectivity, inbound and outbound, is permitted; this policy is distributed to all endpoints, 
which enforce it. In the full-blown version, endpoints are characterized by their IPsec identity, typically in the form of a certificate. Rather than relying on the topological notions of "inside" and "outside", as is done by a traditional firewall, a distributed firewall assigns certain rights to whichever machines own the private keys corresponding to certain public keys. [1][2]

To implement a distributed firewall for allowing and storing authorized and unauthorized specialized database, we need a strong verification and validation security policy language that can describe which connections are acceptable.

\subsection{Basic working of distributed firewalls}

Distributed firewalls are the following three components.

1. A language for expressing policies and resolving requests. In their simplest form, policies in a distributed firewall are functionally equivalent to packet filtering rules. However, it is desirable to use an extensible system (so other types of applications and security checks can be specified and enforced in the future). The language and resolution mechanism should also support credentials, for delegation of rights and authentication purposes .[4]

2. A mechanism for safely distributing security policies. This may be the IPsec key management protocol when possible, or some other protocol. The integrity of the policies transferred must be guaranteed, either through the communication protocol or as part of the policy object description (e.g., they may be digitally signed).

3. A mechanism that applies the security policy to incoming packets or connections, providing the enforcement part.

Distributed firewalls rest on three notions:

- A policy language that states what sort of connections are permitted or prohibited.[3]

- Any of a number of system management tools, such as Microsoft's SMS or ASD, and

- IPSEC, the network-level encryption mechanism for TCP/IP.

\subsection{Components of a distributed}

\section{firewall}

- A management system for designing the policies.

- User specified policy distribution.

- Host Implementation.

\section{Management system}

Management, a component of distributed firewalls, makes it practical to secure enterprise-wide servers, desktops, laptops, and workstations. Central management provides greater control and efficiency and it decreases the maintenance costs of managing global security installations. This feature addresses the need to maximize network security resources by enabling policies to be centrally configured, deployed, monitored, and updated. From a single workstation, distributed firewalls can be scanned to understand the current operating policy and to determine if updating is required.

\section{User specified policy distribution}

The policy distribution scheme should guarantee the integrity of the policy during transfer. The distribution of the policy can be different and varies with the implementation. It can be either directly pushed to end systems, or pulled when necessary. [3]

\section{Host implementation}

The security policies transmitted from the central management server have to be implemented by the host. The host end part of the Distributed Firewall does provide any administrative control for the network administrator to control the implementation of policies. The host allows traffic based on the security rules it has implemented.

\subsection{Policy language}

Policy is enforced by each individual host that participates in a distributed firewall. The distributed firewall administrator--who is no longer necessarily the "local" administrator, since we are no longer constrained by by topology--defines the security policy in terms of host identifiers. The resulting policy (probably, though not necessarily, compiled to some convenient internal format) is then shipped out, much like any other change. This policy file is consulted before processing incoming or outgoing messages, to verify their compliance. It is most natural to think of this happening at the network or transport layers, but policies and enforcement can equally well apply to the application layer.

\subsection{Policy verification}

Policy verification is enforced by the each incoming packet as per the user specified policy and also verifies the inconsistencies.

\subsection{Policy validation}

A policy validation method normally validating firewall security policy in a heterogeneous network with a complex layout.

The policy validation system is concerned; there are two kinds of failure. [13]

Host Failure Any of the network hosts can fail at any time. The things that a node needs to keep track of ongoing tests, previous test results it is possible to store all of that information on Database.. [13] 
Network Failure The network can obviously fail at any time, or can simply not be laid out as expected. From this perspective, any command that gets lost can be viewed as an unexpected, failed network test. These can be ignored or reported to the root Manager in some way, as they indicate a network status that to the distributed firewall administrator. [13]

\subsection{Distributed firewall policy advisor (DFPA)}

In DFPA techniques are simplifies the management of filtering rules and also maintain the strong security of firewalls.

The filtering rules and policy rules are implemented using java programming language in a software tool called DFPA. [6] [7]

\subsection{Specialized database (SDB)}

Database is collection of related data and access those data. The collection of data usually referred to as Database (DB).

The current research propose the specialized database for allowing and storing of authorized and unauthorized database user according to policy verification and validation scheme.

\section{Threat Comparison}

Distributed firewalls have both strengths and weaknesses when compared to conventional firewalls. By far the biggest difference is their reliance on topology. If your topology does not permit reliance on traditional firewall techniques. [5]

\subsection{Service exposure and port scanning}

Both types of firewalls are excellent at rejecting connection requests for inappropriate services. Conventional firewalls drop the requests at the border or edge; distributed firewalls do so at the host. A more interesting question is what is noticed by the host attempting to connect. Today, such packets are typically discarded, with no notification. A distributed firewall may choose to discard the packet, under the assumption that its legal peers know to use IPSEC; alternatively, it may instead send back a response requesting that the connection be authenticated, which in turn gives notice of the existence of the host.

Firewalls built on pure packet filters cannot reject some "stealth scans" very well. One technique, for example, uses fragmented packets that can pass through unexamined because the port numbers aren't present in the first fragment. A distributed firewall will reassemble the packet and then reject it.

\subsection{Application-level proxies}

Some services require an application-level proxy. Conventional firewalls often have an edge here; the filtering code is complex and not generally available on host platforms. As noted, a hybrid technique can often be used to overcome this disadvantage. In some cases, of course, application-level controls can avoid the problem entirely. If the security administrator can configure all Web browsers to reject ActiveX, there is no need to filter incoming HTML via a proxy.

In other cases, a suitably sophisticated IPSEC implementation will suffice. For example, there may be no need to use a proxy that scans outbound FTP control messages for PORT commands, if the kernel will permit an application that has opened an outbound connection to receive inbound connections.

\subsection{Intrusion detection}

Many firewalls detect attempted intrusions. If that functionality is to be provided by a distributed firewall, each individual host has to notice probes and forward them to some central location for processing and correlation. The former problem is not hard; many hosts already log such attempts. One can make a good case that such detection should be done in any event. Collection is more problematic, especially at times of poor connectivity to the central site. There is also the risk of co-coordinated attacks in effect causing a denial of service attack against the central machine.

\subsection{Insider attacks}

At first glance, the biggest weakness of distributed firewalls is their greater susceptibility to lack of cooperation by users. Although there are technical measures that can be taken, as discussed earlier, these are limited in their ability to cope with serious misbehavior. That said, we assert that this problem is not a real differentiator. Even conventional firewalls are easily subverted by an uncooperative insider. In other words, an insider who wishes to violate firewall policy, the firewall administrator filter that packet.

On the other hand, distributed firewalls can reduce the threat of actual attacks by insiders, simply by making it easier to set up smaller groups of users. Thus, one can restrict access to a file server to only those users who need it, rather than letting anyone inside the company pound on it.

\section{IMPLEMENTAION TECHNIQUES}

\subsection{Use case diagram}

A use case is an interaction between users and a system; it captures the goal of the users and the responsibility of the system to its users. The current research in our implementation techniques diagrammatic representation as follows 


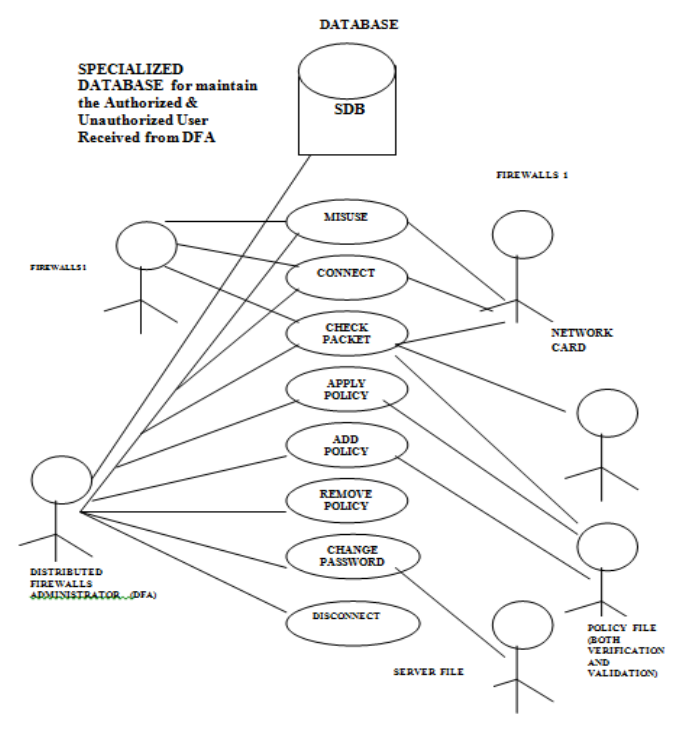

system and its environment.

\section{List of actors in the distributed firewall}

- $\quad$ Add policy

- Remove policy

- Apply policy

- Connect

- Disconnect

- Change password

- Misuse

- $\quad$ Check packets

\section{Add policy}

The distributed firewall administrator adds the policy to the firewall, which is stored in the temporary file.

\section{Remove policy}

The distributed firewall administrator removes the policy from the firewall, which is stored in the temporary file.

\section{Apply policy}

The distributed Firewall administrator updates the policy of the firewall from the temporary file.

\section{Connect}

Distributed firewall administrator to connect the system.

\section{Successful case}

Distributed firewall administrator makes a request control from the firewall, the control is granted.

\section{Failure case}

Firewall administrator makes a request to the firewall, as there is no firewall request gets timeout.

\section{Disconnect}

Distributed firewall administrator change to the new password by giving the old password and the new password.

\section{Misuse}

Firewall gives the blocked details to the firewall administrator which is stored in the misuse file and that can be viewed by the firewall administrator.

\section{Check packet}

Firewall checks the packets as per the user the policy.

\section{RELATED WORK:}

Current research on distributed firewall for authorized and unauthorized database user according to the policy verification and validation mainly focus the following.

1. Maintaining the database for both authorized and unauthorized (ie.collecting the information from distributed firewall administrator).

2. Verifying and validating the security policy in the networks.

3. The testing and validating firewalls regularly.

4. Identify the Static and dynamic vulnerability analysis.

5. Strong Authentication and Authorization for each firewalls.

\section{CONCLUSION:}

The main objective of this research is to implement a authorized and unauthorized database user according to the policy verification and validation of distributed firewall under the specialized database(SDB).In distributed firewall environment in order to keep track of some certain actions in the first stage (Create, Read, Update, Delete) that are performed on the policy rule set. Then distributed firewall concept is explained and the comparison of two firewall designs is presented in terms of their performance in network security. The next stage is to give the details of distributed firewall environment for which the proposed the maintain specialized database is designed. Such an application will be very helpful in network security management in protecting the consistency among the overall security policy. The data provided by the application can be used to implement more advanced tools like distributed firewall policy advisor tools(DFPA).

\section{REFERENCES:}

[1] G. Yan, S. Chen, and S. Eidenbenz. Dynamic balancing of packet filtering workloads on distributed firewalls. Technical Report LA-UR- 07-3281, Los Alamos National Laboratory, 2007.

[2] L. Yuan, J. Mai, Z. Su, H. Chen, C. Chuah, and P. Mohapatra. Fireman A toolkit for firewall modeling and analysis. In Proceedings of IEEE Symposium on Security and Privacy, May 2006. 
[3] E. Al-Shaer, H. Hamed, R. Boutaba, and M. Hasan. Conflict classification and analysis of distributed firewall policies. IEEE JSAC, 23(10), October 2005.

4] .Blaze, J.Feigenbaum, J.Loandis and A.Keroymtis.The role of Trust management in Distributed systems security.In Secure Internet programming [20], pages 185-20.

[5] Frank Swiderski and Window Snyder. Threat Modeling. Microsoft Press, 2004.

[6] E. Al-Shaer and H. Hamed. "Firewall Policy Advisor for Anomaly Detection and Rule Editing." IEEE/IFIP Integrated Management Conference (IM'2003), March 2003.

[7] E. Al-Shaer and H. Hamed. "Design and Implementation of Firewall Policy Advisor Tools." DePaul CTI Technical Report, CTI-TR-02-006, August 2002.

[8] D. Chapman and E. Zwicky. Building Internet Firewalls, Second Edition, Orielly \& Associates Inc., 2000.

[9] D. Eppstein and S. Muthukrishnan. "Internet Packet Filter Management and Rectangle Geometry." Proceedings of 12th Annual ACM-SIAM Symposium on Discrete Algorithms (SODA), January 2001.
[10] A. Mayer, A. Wool and E. Ziskind. "Fang: A Firewall Analysis Engine." Proceedings of 2000 IEEE Symposium on Security and Privacy, May 2000.

[11] S. Ioannidis, A. D. Keromytis, S. M. Bellovin and J. M. Smith, "Implementing a Distributed Firewall", ACM Conference on Computer and Communications Security, Athens, Greece, November 2000.

[12] S. M. Bellovin, "Distributed Firewall",; login: magazine, Special issue on Security, November 1999.

[13]. Kyle Wheeler. Distributed firewall policy validation, December 7, 2004

\section{AUTHORS}

1. P.SENTHILKUMAR is working as Lecturer in Computer Science and Engineering, Nandha College of Technology at Erode. He received his M.E. (CSE) in 2008 from Anna University, Chennai. Pursing Ph.D in Anna University-Coimbatore, TamilNadu.His areas of research interests include Networks and Network Security. He has published two International Conferences.

2. DR.S.ARUMUGAM is an Chief Executive Officer of Nandha Engineering College, Erode. He has Guided various Research scholar. 